\title{
Terapia acuática en neurorrehabilitación
}

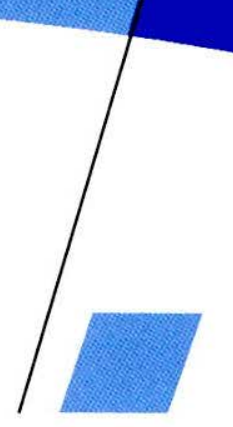

Fabiola Moscoso Alvarado*

\section{Resumen}

La terapia acuática es muy útil para mejorar la función de las personas en inmersión, porque en el agua los pacientes flotan fácilmente, propiciando mejor movimiento con menor esfuerzo, la resistencia causada por la viscosidad del agua, le ayuda al fortalecimiento muscular; la presión que el fluido ejerce simultáneamente en todo el cuerpo inmerso (presión hidrostática) disminuye el edema, incrementa el gasto cardiaco, aumenta la presión intratoráxica, durante el ejercicio. El agua tibia, aumenta la temperatura del tejido superficial creando un efecto sedativo, reduce la tensión muscular secundaria, favorece el flujo sanguíneo, restaura la oxigenación de los tejidos, y remueve los desechos, mejora la nutrición, disminuye la rigidez y el dolor inducido por la isquemia. Además, acelera las funciones metabólicas de las células, la circulación de la sangre y la linfa. Por esta razón un trabajo efectivo debe incluir actividades que permitan vivenciar los principios físicos de la mecánica de fluidos y técnicas de tratamiento fisioterapéutico.

Palabras clave: terapia acuática, mecánica de fluidos, resistencia, rigidez.

\section{Abstract}

The acuatic therapy is very useful to improve activity of people in immersion, as patients float easily in the water, producing better movement with less effort, and the resistance caused by the water viscosity helps to strength muscles; the pressure from fluids on the whole body (hydrostatic pressure) decreases edema, increases cardiac output, increases intra thoracic pressure, during exercise. Warm water, increases the temperature of superficial tissue creating a sedative effect, reducing secondary muscular tension, improving blood flow, restoring tissue oxygenation, removing wastes, improving nutrition, decreasing rigidity and pain due to ischemia. Moreover, it speeds up cell metabolic functions, blood and lymph flow. So, an effective work must include activities to experience physical principles of fluids mechanics and physiotherapeutic treatment.

* Fisioterapeuta Universidad Nacional de Colombia, terapeuta neurodesarrollista, terapeuta acuática clínica universitaria Teletón. 


\section{Introducción}

Dentro de la gran variedad de modalidades utilizadas por los fisioterapeutas para el manejo de las diversas patologías, tiene cada vez mayor reconocimiento y aceptación la hidroterapia la cual se deriva de las palabras griegas hydor: agua y therapia: curación. Es una modalidad terapéutica que utiliza el agua a través de equipos y elementos para el tratamiento de los pacientes con diversas patologías, en otras palabras, es la aplicación de un agente físico sobre tejidos biológicos con el fin de obtener cambios terapéuticos (Mogollón, 2005).

Como parte esencial de la hidroterapia la terapia acuática se define como un procedimiento y no una modalidad, y es empleada para mejorar la función mediante la aplicación de técnicas por parte de un fisioterapeuta con el paciente en inmersión.

A nivel mundial la terapia acuática ha sido utilizada a través de los siglos en piscinas minerales, ríos, océanos, en forma pasiva inicialmente y posteriormente con técnicas desarrolladas a través de los años al descubrirse los beneficios del agua tibia y la activa participación del paciente. En los años cincuenta tiene un declive en su interés principalmente en los centros médicos y resurge hacia los años setenta aunque la demanda en hospitales era limitado por su alto costo. A partir de los años ochenta toma la mayor fuerza, se desarrolla y fortalece su sustento teórico y algunas de las técnicas lo que permite una mayor aceptación a todo nivel. (Hejar y Blunt, 2006). En el siglo XXI se entiende que es una necesidad y no un lujo, por lo que en todos los países industrializados y algunos del tercer mundo ya se tienen establecidos centros y programas a todo nivel.

En Colombia el desarrollo de la terapia acuática ha sido lento teniendo como principal enemigo el factor económico. Algunas instituciones han mantenido sus programas enfatizando principalmente en el ejercicio acuático y no en terapia acuática. En el año 2001 la Clínica Universitaria Teletón inaugura el área de Hidroterapia más moderna del país y de Suramérica, la cual cuenta con tecnología de punta. Donde se incluye una piscina y un tanque de marcha de piso ajustable. Como parte de este proceso se realizó una capacitación en hidroterapia y terapia acuática con el terapeuta acuático Johan Lambeck quien tiene amplia trayectoria y reconocimiento a nivel mundial. A partir de entonces la clínica ha atendido anualmente a numerosos pacientes con patología neurológica dentro del programa integral de rehabilitación (PIR), además de ser pionera en el desarrollo de programas de capacitación en terapia acuática para fisioterapeutas de fuera de Bogotá.

Para entender los beneficios de la terapia acuática es necesario primero conocer las bases físicas y fisiológicas sobres las cuales se basa está modalidad de intervención. A continuación se revisarán aspectos relacionados con la mecánica de fluidos y sus efectos a nivel fisiológico.

\section{Mecánica de fluidos}

Es la parte de la física que se ocupa de la acción de los fluidos en reposo o en movimiento; conocer y entender los principios básicos de la misma es esencial en el análisis y diseño de cualquier sistema en el cual el fluido es el elemento de trabajo (http:/ www.monografias.com/trabajos 12/mecfluidos/ mecflui.shtml), como lo es el agua desde el punto de vista de la terapia acuática.

Los fluidos, como todos los materiales, tienen propiedades físicas que permiten caracterizar y cuantificar su comportamiento así como distinguirlos de otros. Para el tema que se está abordando se va a revisar la densidad relativa, la viscosidad y la tensión superficial como propiedades importantes del agua.

\section{Densidad relativa}

Es la relación entre la masa y el volumen de determinado objeto, la cual se mide en $\mathrm{kg} / \mathrm{m}$. En el agua se utiliza el término densidad relativa y no tiene unidad, esta propiedad determina si un objeto puede o no flotar en el agua. La densidad relativa del agua es $0.995-0.999$ mientras que la del aire es 0.0013 
casi unas 800 veces menos; el cuerpo tiene una densidad entre $0.95-0.99$, la densidad del tejido adiposo es $0.90-0.94$ mientras que el tejido óseo 1.40 - 1.80 (Lambeck, 2005). Para que un cuerpo flote en el agua su densidad relativa debe ser menor a la del agua, es por eso que en el cuerpo humano el tórax tiende a flotar mientras que los miembros inferiores a hundirse. En los pacientes que tienen alteración en la densidad normal de sus extremidades vemos las tendencias a flotar en casos de disminución o hipotonía y al hundimiento cuando se presenta hipertonía.

\section{Viscosidad}

Es el tipo de fricción que tiene lugar entre las moléculas de un líquido y produce una resistencia al flujo del mismo. Esta fricción expresa la viscosidad con la que el líquido fluye y, por lo tanto, sólo se aprecia cuando el fluido está en movimiento. La viscosidad actúa como resistencia al movimiento, puesto que las moléculas de un líquido tienden a adherirse a la superficie de un cuerpo que se mueva en él (Panneso y Moscoso, 1999). En el cuerpo humano se presenta mayor resistencia al movimiento por lo que se genera un mayor gasto energético aeróbico, también permite un fortalecimiento muscular equilibrado, incremento en la activación muscular en el tronco, lentificación de los tiempos para las reacciones de enderezamiento y equilibrio en el incremento de las reacciones sensoriales (Mogollón, 2005).

\section{Tensión superficial}

Es la fuerza que se ejerce entre las moléculas de la superficie de un fluido. Actúa como una resistencia al movimiento cuando una extremidad es sumergida parcialmente, pues la tensión superficial ha de romperse por el movimiento (Panneso y Moscoso, 1999).

La mecánica de fluidos puede subdividirse en dos campos principales: la estática de fluidos, o hidrostática, que se ocupa de los fluidos en reposo, y la dinámica de fluidos, que trata de los fluidos en movimiento. Dentro de la estática de fluidos o hidrostática están el principio de Pascal y el de Arquímedes:

\section{Presión hidrostática}

Esta propiedad es explicada por el principio de Pascal, el cual establece que "la presión del fluido se ejerce por igual en toda la superficie de un cuerpo inmerso en reposo en la profundidad dada". La presión se incrementa linealmente con la profundidad del agua y con la densidad en $1 \mathrm{~mm} \mathrm{Hg}$ por $1.36 \mathrm{~cm}$ (Schoedinger, 2000).

Son varios los efectos fisiológicos que por este principio se producen, a nivel circulatorio donde hay una redistribución del flujo sanguíneo desde las partes inmersas hacia el tórax causando un aumento del $60 \%$ en el retorno venoso central con todos los efectos que esta situación genera, disminución del edema, incremento del gasto cardiaco, aumento de la presión intratoráxica, bradicardia durante el ejercicio comparado con tierra.

A nivel pulmonar se presenta una disminución de la capacidad vital entre el 5-10\% por disminución en los volúmenes pulmonares y la resistencia ejercida sobre la musculatura inspiradora, razón por la cual es una contraindicación el ingreso en inmersión a personas con capacidad vital menor a $1500 \mathrm{ml}$ (Hall, Bisson \& Hare).

A nivel renal se produce supresión del sistema reninaangiotensina-aldosterona, disminución de la hormona antidiurética, que a su vez: favorece el incremento en la producción de orina de hasta $7 \mathrm{ml}$. por minuto, disminución del volumen del plasma, movilización del fluido extracelular, incremento en la pérdida de sodio y potasio y supresión de la sed (Mogollón, 2005).

\section{Flotación}

La base de esta propiedad física se halla en el principio de Arquímedes según el cual cuando un cuerpo está total o parcialmente inmerso en un líquido en reposo, experimenta un empuje hacia arriba igual al peso del volumen del líquido desplazado. Es una 
propiedad que brinda muchas herramientas al terapeuta ya que le permite trabajar en forma asistida, como soporte, o en forma resistida (http:/ terapeuticasacuaticas.com/index.html). Cuando el cuerpo humano está inmerso en el agua experimenta dos fuerzas contrarias la de flotación y de la gravedad las cuales deben encontrarse alineadas pero en direcciones contrarias y además deben tener la misma magnitud, para lograr equilibrio del cuerpo. Cuando esto no es así se produce una rotación conocida como el efecto metacéntrico (Lambeck, 2005).

La flotación tiene su mayor influencia en el sistema musculoesquelético generando disminución del soporte de peso y de las fuerzas de compresión a nivel articular, también se presenta alguna inhibición de los músculos espásticos debido probablemente a la disminución de la actividad de las fibras gamma las cuales disminuyen la actividad del huso neuromuscular (Panneso y Moscoso, 1999).

El otro campo de la mecánica de fluidos es la dinámica de fluidos o hidrodinámica que se ocupa de los fluidos en movimiento para lo cual se tiene en cuenta el tipo de fluido:

El Teorema de Bernoulli afirma que la energía mecánica total de un flujo incomprensible y no viscoso ( $\sin$ rozamiento) es constante a lo largo de las líneas de corriente. Este teorema relaciona los efectos de la presión, la velocidad y la gravedad e indica que la velocidad aumenta cuando la presión disminuye (Barsuto, 2006).

Osborne Reynolds demostró la existencia de dos tipos de flujo viscoso en tuberías. A velocidades bajas, las partículas del fluido siguen las líneas de corriente (flujo laminar), y los resultados experimentales coinciden con las predicciones analíticas. A velocidades más elevadas, surgen fluctuaciones en la velocidad del flujo, o remolinos (flujo turbulento), en una forma que ni siquiera en la actualidad se puede predecir completamente (Barsuto, 2006). Estos flujos son de gran importancia y aplicación en la terapia acuática y los efectos a nivel fisiológico están dados de acuerdo a la utilización que de ellos se haga como elementos de intervención terapéutico.

Por último como un principio de gran aplicabilidad en el campo de la hidroterapia está la transferencia térmica, ya que el agua es un conductor efectivo de calor, lo transfiere 25 veces más rápido que en el aire.

La capacidad del calor específico del agua es de 1. El cuerpo humano en el agua puede ganar o perder calor muy rápidamente dependiendo de la temperatura del agua (Mogollón, 2005). Según lo reporta Poteat la inmersión en agua tibia (por encima de la termoneutral $34-35^{\circ} \mathrm{C}$ ) aumenta la temperatura del tejido superficial creando un efecto sedativo, hay inhibición en la activación de las fibras motoras gamma lo que disminuye el estiramiento del huso neuromuscular, se eleva la temperatura del huso neuromuscular y del órgano tendinoso de Golgi que disminuyen su rata de actividad. Por otro lado hay reducción en la tensión muscular secundaria, favoreciendo el flujo sanguíneo lo que restaura la oxigenación normal de los tejidos y remueve los irritantes químicos (desechos) lo que tiene como efecto mejorar la nutrición, disminuir la rigidez y disminuir el dolor inducido por la isquemia.

También por la estimulación de los receptores térmicos se disminuye la percepción (no-sensación) de dolor durante la estimulación directa de estos receptores. Por último hay aceleración de las funciones metabólicas de las células, la circulación de la sangre y la linfa. Esto incrementa la oxigenación de músculos isquémicos y promueve la eliminación de la irritación química de desechos, los que disminuyen la isquemia de los músculos y la toxemia y por tanto disminuye la sensación dolorosa. (Poteat, 1997).

\section{Beneficios de la terapia acuática en neurorrehabilitación}

La utilización de la terapia acuática en el campo de la neurorrehabilitación se sustenta cada día en nu- 
merosas investigaciones que se han realizado en las cinco últimas décadas y que han dado la base teórica para la evolución de los diferentes enfoques y técnicas de intervención en el área.

Al revisar estas investigaciones se logra determinar cuáles son los efectos más significativos que serán presentados a continuación:

\section{Modulación del tono anormal}

El primer sustento para este efecto lo brindan los estudios de Harrison y colaboradores quienes determinaron que un cuerpo en inmersión con el agua a la altura de la apófisis xifoides experimenta una descarga de peso entre $25-37 \%$ del total de su peso en tierra (Harrison \& Bulstrode, 1987), si camina lentamente de un 50-75\% menos y al caminar rápidamente un $75 \%$ del peso total (Harrison, Hillma y Bulstrode, 1992). Por esta razón cuando un paciente está en inmersión maneja un peso menor, existe menor fuerza gravitacional lo que disminuye la necesidad de utilizar cocontracción anormal tal y como se ve en tierra.

Adicionalmente según Schoedinger debido a la transferencia térmica en un medio tibio, se obtiene una disminución de la actividad de las fibras gamma, lo que se ve reflejado en una disminución en el esfuerzo requerido para el movimiento voluntario favoreciendo así movimientos de mayor complejidad (Schoedinger, 2000).

Finalmente, el trabajar en un medio líquido donde hay un movimiento continuo produce un incremento en la estimulación vestibular que conlleva una disminución en el tono muscular, especialmente en aquellas técnicas de terapia acuática en donde se realizan movimientos rotacionales del tronco, movimiento rítmico y repetitivo (Schoedinger, 2005). Cabe anotar que la utilización de movimientos rápidos favorece el aumento del tono muscular en casos de hipotonía (Stuchiner, 2000).

\section{Disminución de la sensación dolorosa y mejoría en rangos de movimiento}

Según Schoedinger se pueden mejorar rangos de movimiento tanto pasivos como activos (Schoedinger, 2000); la mejoría en los pasivos se sustenta en los estudios de Bovy citados por Lambeck, que muestran cómo la inmersión en agua tibia afecta la viscoelasticidad del tejido conectivo en el músculo proveyendo mayor nutrición y oxígeno debido al aumento de la circulación y mejorando la reabsorción de desecho metabólicos (Bovy, 2001). Este aspecto es muy favorable para la realización de movilizaciones, estiramientos y desbloqueos que van a ayudar a lograr relajación local y general facilitando ganancia en rangos de movimiento, flexibilidad y disminución de la sensación dolorosa (Lambeck, 2001). Los rangos activos se mejoran debido a que la flotación asiste no sólo el movimiento a través de la superficie del agua, sino que también soporta el movimiento paralelo a ésta. Por otro lado los programas activos de terapia acuática se han utilizado para incrementar el rango de movimiento (Schoedinger, 2005.

\section{Mejoría en el balance}

Al revisar las bases sobre las cuales se desarrolla el trabajo de balance en agua se encuentran opiniones como las de Aizpurviete (Aizpurviete, 1997) y Poteat (Poteat, 2006) quienes mencionan que los pacientes con problemas de balance frecuentemente tienen un gran miedo a caer. En el agua, con el calor y la flotación, se obtiene un medio óptimo, seguro y confortable para iniciar los ejercicios de balance.

Por otro lado, Poteat menciona cómo la viscosidad actúa como resistencia al movimiento, previene una rápida caída y aumenta el período de tiempo en el cual el paciente puede reaccionar para corregir más fácilmente sus errores, al igual que para aumentar el rango en el movimiento del centro de gravedad sobre la base de soporte ya que mejoran sus reacciones de enderezamiento y equilibratorias sin que esto tenga consecuencias traumáticas, obteniéndose ma- 
yor seguridad, independencia y confianza para el paciente (Poteat, 2006).

Poteat hace referencia a un trabajo de McNeal en el que afirma que debido a la resistencia distal que se produce en las extremidades durante el movimiento en el medio acuático, hay una respuesta de mayor cocontracción de los músculos del tronco que incrementa su control y el tiempo de respuesta en las reacciones de equilibrio del paciente (McNeal, 1997).

El agua, a pesar de ser un medio inestable, brinda una adecuada estimulación al paciente con problemas de balance. Al trabajar el control del balance desde el punto de vista del enfoque Halliwick, se controlan los límites de la estabilidad y se le brinda el soporte necesario al paciente, para desempeñarse en un medio seguro y muy beneficioso para su problema (Gresswell y Maes, 2000).

\section{Favorece la realización de actividades funcionales}

Los pacientes pueden realizar movimientos específicos asistidos por flotación con menor esfuerzo que en tierra, igualmente logran ejecutar actividades con incremento gradual en la complejidad, especialmente aquellas que involucran actividades con movimientos recíprocos y con retos para el balance

Por otro lado y debido a la flotación y la viscosidad, los pacientes pueden asumir posturas verticales y realizar marcha, mucho antes de empezarlo a hacer en tierra (Lambeck, 2001). Igualmente los mecanismos y fases de la marcha se ven favorecidos observándose mayor calidad, aunque algunos en pacientes con déficit propioceptivo se altera la preparación y colocación del pie para el contacto inicial (Moscoso, 2005).

El impacto psicológico que tiene en los pacientes el hecho de lograr la realización de un número mayor de actividades, incluso llegando a ser independientes, es un factor determinante para su autoestima y motivación, tanto para trabajar en el medio acuático, como por las implicaciones en su vida diaria al sentirse capaces de realizar algunas cosas en forma independiente (Moscoso, 2005).

\section{Mejoría en fuerza y resistencia muscular y aeróbica}

Debido al desacondicionamiento físico resultante de la discapacidad, los ejercicios de fortalecimiento y los aeróbicos son vitales en el tratamiento de personas con desórdenes neurológicos y el agua es un medio donde lo puede hacer con mayor seguridad debido a que disminuye la descarga de peso y por lo tanto, reduce la posibilidad de síndromes por sobreuso (Torpe y Reilly, 2000).

\section{Mejora la estimulación sensorial}

A nivel propioceptivo, Poteat, en una conferencia sobre el reentrenamiento propioceptivo con terapia acuática, refiere cómo la presión hidrostática puede estimular receptores de presión y dar una retroalimentación propioceptiva durante la ejecución del ejercicio (Poteat, 1997). Otros autores mencionan el aumento en la estimulación vestibular y su influencia en el tono y los ajustes posturales (Schoedinger, 2005).

\section{Enfoques de intervención en terapia acuática}

Los enfoques de intervención en terapia acuática más conocidos y con mayor aplicación para los pacientes neurológicos son el Concepto Halliwick y el Método Bad Ragaz.

\section{Concepto Halliwick}

Fue diseñado y desarrollado en 1950 por James McMillan, un ingeniero, experto en mecánica de fluidos y profesor de natación. El objetivo inicial fue crear un programa con el objetivo de enseñar a nadar a personas con discapacidad, pero desde 1975 el método se ha extendido a la rehabilitación acuáti- 
ca a través del llamado "Enfoque lógico del ejercicio en agua" mostrando su potencial en los campos pediátrico, neurológico, reumatológico y ortopédico (Moscoso, 2004). Con el paso del tiempo los principios se han profundizado y renovado a tal punto que hoy en día es el método más usado en rehabilitación neurológica y es difundido por el mundo como Concepto Halliwick. Se basa en un sistema de aprendizaje motor donde el control postural es el objetivo más importante.

\section{El programa diseñado por McMillan es el de Diez}

Puntos el cual es una secuencia de aprendizaje motor focalizada en control postural, con el fin de hacer independiente al individuo a través de la natación como forma de desplazamiento. Esta propuesta es el esqueleto para desarrollar el "Enfoque lógico del ejercicio en agua" o los "Ejercicios específicos en agua", que es una estructura que ayuda a planear, desarrollar y ejecutar un programa, a la vez que se toman las decisiones clínicas en las sesiones de terapia acuática (Moscoso, 2004).

El Programa de Diez Puntos se divide en tres fases: la adaptación mental, el control del balance y el movimiento. Estas etapas se pueden sobreponer una a la otra sin que sea necesario que se dominen totalmente, aunque se debe mantener el orden para evitar el error de tratar de enseñar movimientos complejos de natación a una persona que tiene en su mente únicamente el objetivo de sobrevivir (Nicol, Schmidt-Hansberg y McMillan, 1979). Todas las etapas se deben dominar para ser competente en agua (Gresswell y Maes, 2000).

La adaptación mental es un proceso en el que gradualmente se le va dando menor soporte al paciente de acuerdo a las habilidades que vayan desarrollando. Dentro del ajuste mental se halla el desapego, que es un proceso continuo de cambios en el soporte usando elementos hidrodinámicos para incrementar la dificultad y retar la estabilidad y es utilizado en todas las etapas cuando se introducen nuevas habilidades. Su objetivo final es lograr una independencia física y mental del paciente IHA, 2000 citado por
Gresswell, Maes, (Gresswell y Maes, 2000). Los aspectos que se tienen en cuenta para el despego son: cambio en el soporte, la mecánica de fluidos, la mecánica, la fisiología, el aprendizaje motor y algunos tópicos generales (Lamberck, 2001).

En esta etapa es importante el control respiratorio ya que facilita movimientos hacia adelante lo cual es esencial para actividades de balance en agua. También favorece el sellado labial, la vocalización y las actividades diafragmáticas.

La segunda fase es el control del balance la cual es una habilidad para mantener o cambiar en forma independiente una posición en el agua, se debe desarrollar un grado de control de balance automático y centralizado para evitar movimientos indeseados y lograr un eficiente control postural. Las etapas que componen esta fase son: los controles rotacionales en diferentes ejes, la inversión mental, el balance estático y el deslizamiento con arrastre.

En la primera etapa se trabajan los controles rotaciones sagitales, transversales, longitudinales y combinados para lograr estabilidad postural.

La rotación sagital se realiza en posición vertical inclinando el cuerpo de derecha a izquierda o descargando peso a cada lado, esto permite aumentar los rangos de movimiento y estabilidad en columna al igual que favorece las reacciones de enderezamiento y protectivas laterales.

La rotación transversal se realiza alrededor del eje transversal con la persona moviéndose desde bípedo a supino o prono y viceversa, es un tipo de disociación o extensión selectiva donde todos los componentes de la cadena pueden ser ejercitados buscando en todo momento la simetría.

La rotación longitudinal se realiza alrededor de la columna produciendo un rolado de $360^{\circ}$ donde se favorece las reacciones de enderezamiento entre cabeza, cintura escapular, y cintura pélvica con un gran trabajo por parte de los oblicuos actuando como estabilizadores. Este control es el que mayor reper- 
cusión tiene en actividades funcionales como al nadar y caminar.

La rotación combinada se realiza combinando los movimientos en los ejes sagital con longitudinal o trasversal con longitudinal y es utilizada para enseñar el concepto de rolar fuera del problema, colocando la cara hacia la superficie; esta rotación genera movimientos en tres dimensiones (Lamberck y Coffey, 2000).

Después que la persona maneja perfectamente estos controles se introduce la inversión mental, la cual se basa en el principio hidrodinámico de la flotación donde cualquier cuerpo sumergido tiende a salir a la superficie, la persona debe utilizar alguno de los controles y llegar a la superficie en una posición que pueda respirar.

Estas cinco primeras etapas requieren una cantidad máxima de movimiento, una vez son manejadas completamente se introducen los aspectos estáticos del programa donde se requiere un grado fino de control postural centralizado; la persona asume diversas posiciones y se introducen variables como la turbulencia o la estimulación táctil para generar inestabilidad, se debe responder con control estático.

Una vez se consigue la estabilidad estática se introduce el movimiento con arrastre, donde la persona es arrastrada con el movimiento del instructor, durante este proceso se produce turbulencia manual debajo de la persona y la cual debe mantener una posición del cuerpo estable y balanceada sin incrementar el radio, el tono o chapotear. Esto permite a la persona experimentar un control postural centralizado mientras es arrastrado a través del agua.

La tercera fase es la de movimiento, que tiene las etapas de la progresión simple, y los movimientos básicos de natación. En este punto la persona es capaz de crear movimientos efectivos, eficientes y hábiles en el agua. Después que la persona controla el movimiento producido por el arrastre se le introducen movimientos simples para que sea capaz de moverse independientemente en el agua de acuerdo a sus condiciones particulares. Generalmente se inicia en posición supino con movimientos simétricos de ambos brazos. Al nadar se requiere de una coordinación central propia y sincronismo por lo que esta fase tiene como propósito terapéutico el entrenamiento dinámico de la estabilidad del tronco.

Como se mencionó anteriormente el desarrollo de estos puntos son el esqueleto para el enfoque terapéutico de la sesión. Según Lamberck, (Lamberck, 2001 ) entre las muchas opciones que se tienen al desarrollar una sesión de tratamiento siempre se deben considerar los objetivos, los planos de rotación, el modelo (preentrenamiento, inhibición, facilitación y ejercicios dinámicos), los patrones de ejercicio, la posición inicial, la profundidad del agua en el paciente y la técnica del tratamiento.

Al realizar un enfoque holistico dentro de la sesión se busca mejorar las áreas sensorial, motora, cognitiva y perceptual, las cuales tienen a su vez un interés terapéutico definido a cada paciente (Gresswell y Maes, 2000).

\section{Método de Bad Ragaz}

Fue desarrollado inicialmente en Alemania hacia 1950. Posteriormente, en 1957, se introduce como parte del tratamiento de pacientes en el centro de salud de Bad Ragaz, Suiza de donde toma su nombre (Mogollón, 2005).

Comenzó como una forma de fortalecer los músculos a través de un patrón unidimensional simple y fue progresando hasta convertirse en un método en el que el terapeuta actúa como punto de apoyo en la cadena cinética para producir patrones tridimensionales de movimiento a través del agua. La aplicación de estos patrones toma como referencia los principios de la facilitación neuromuscular propioceptiva y con ello la combinación de una serie de contracciones isométricas e isotónicas cuya resistencia se gradúa de acuerdo a las capacidades del paciente. 
Este método utiliza las propiedades físicas del agua -flotación, viscosidad y presión hidrostática - para facilitar las funciones fisiológicas y anatómicas de las articulaciones y los músculos. Es un método de reeducación, fortalecimiento, relajación, estiramiento muscular e inhibidor del tono anormal (Gaviria, 2004) y con el cual se puede lograr aumento del rango articular, relajación, mejoría en la alineación corporal y estabilidad del tronco, mejoramiento de la coordinación de los patrones normales de movimiento, disminución del dolor, tracción y elongación espinal, mejoramiento de las habilidades funcionales y preparación de los miembros inferiores para el soporte de peso.

Para su aplicación se requiere de la utilización de ayudas de flotación las cuales ofrecen seguridad y ayudan a estabilizar al usuario en el agua. Los flotadores no deben restringir el movimiento. Se deben tener ayudas de flotación en cuello y pelvis principalmente, usar collar cervical en cuello y cinturón pélvico a la altura de S2 asegurándose de obtener una posición neutra, el cuello y la pelvis deben quedar alineados, se debe evitar que produzcan hiperflexión o hiperextensión. Opcionalmente pueden colocarse en piernas y brazos según se necesite, es preferible que sean ajustables, pues así se puede variar la resistencia. No es necesario que tengan mucho aire. El equipo debe ser seguro y confortable, debe ser apropiado teniendo en cuenta el tamaño y la densidad de la persona (Lambeck, 2001).

La posición del terapeuta debe ser estable, con el nivel del agua debe estar a nivel de T8 - T11 para evitar la curva de pánico del balance en agua que le genera inestabilidad y más bajo le puede ocasionar problemas en la espalda por adquirir una posición inadecuada. Debe ubicarse en la línea diagonal del movimiento, debe observar todo el tiempo el movimiento (Lamberck y Coffey, 2000).

De acuerdo a Reid-Campion hay tres tipos de actividad muscular identificadas en Bad Ragaz: la isométrica donde se tiene a la persona en una posición fija mientras es movida por el terapeuta a través del agua, se utiliza contra resistencia lo que crea contracción muscular.

En la isotónica el grado de resistencia es controlado por el terapeuta quien es el punto estable pero se mueve con el usuario a través del agua aumentando la resistencia durante el movimiento activo y empujado o halando al paciente en la dirección del movimiento.

La isokinética donde el grado de resistencia es dado por el usuario, siendo el terapeuta el punto estabilizador y el usuario se mueve alrededor de él. La resistencia en este movimiento la da la velocidad de la persona a través del agua (ReidCampion, 1997).

La resistencia es brindada por las fuerzas hidrodinámicas. La velocidad con la que se mueve el usuario en el agua y el arrastre que tenga, son factores que intervienen también. Este arrastre es proporcional a la velocidad de la persona; por lo tanto, la resistencia aumenta con la velocidad (Gaviria, 2004).

Los patrones de movimiento se dividen en tres grupos: los patrones que trabajan el cuerpo a través de los miembros inferiores, los patrones que trabajan el cuerpo a través de los miembros superiores y los patrones que trabajan el cuerpo a través del tronco. También se dividen en patrones unilaterales y patrones bilaterales los que a su vez se subdividen en simétricos y asimétricos. Todos los patrones de movimiento se aplican con la persona en supino o en posición rotada.

Haciendo paralelo a la técnica de Rabat, en el método Bad Ragaz existen numerosos patrones de movimiento con sus respectivas variaciones que se establecen de acuerdo a las necesidades y condiciones particulares de cada paciente.

La evaluación del paciente en terapia acuática se divide en dos áreas principalmente: la evalua- 
ción subjetiva y la objetiva, las cuales deben integrarse para un análisis global del tratamiento.

La evaluación subjetiva es lo que el paciente refiere de su condición y en la cual se observa la actitud del paciente en el medio, se comentan sus antecedentes y sus habilidades.

En la evaluación objetiva se describe lo que el terapeuta observa en cuanto al ajuste mental, el control respiratorio, como es su forma y densidad para lo cual se tiene en cuenta la clasificación de Reid-Campion.

Entre las formas puede encontrarse la normal y las formas alteradas como lo son el cuadrilátero desigual, la forma triangular, la forma en silla de ruedas, la forma en tijera, la forma en extensión y el alto centro de flotación. Otros puntos que se evalúan son el control del balance basado en los controles rotacionales de Halliwick, el balance estático en supino y bípedo al igual que el balance dinámico y la capacidad de desplazarse (Lambeck, 2001).

Finalmente se resalta que la evaluación se debe hacer inicialmente y en diferentes momentos del tratamiento. Inclusive se recomienda hacer seguimiento del paciente una vez se haya terminado el tratamiento.

Recibido: julio 2006 Aceptado: septiembre 2006

\section{Referencias bibliográficas}

Aizpurviete, S. (1997). Program Book Aquatic Therapy Symposium 1997. "Balance and Aquatic Therapy". Las Vegas. H58-H161.

Barsuto, L. (2006). Mecánica de fluidos. www.geocities.com/jalarab/cap 7 html.35K Copyright 2000-2001. Acceso febrero.

Bovy, J. citado por LAmbeck, J. (2001). Hydrotherapy in Adult Neurology. Memorias Capacitación Hidroterapia Bogotá, 2.
Fisioterapia acuática. Indicaciones y tiempos de recuperación http://www.terapeuticasacuaticas. com/index.html Julio de 2006.

Gaviria, F. (2004). El método Bad Ragaz. Memorias Simposio de Actualización en Hidroterapia. Clínica Universitaria Teletón.

Gresswell. A., Maes. JP. (2000). Principles of Halliwick and its application for children and adults with neurological conditions. Presentation for the HACP workshop Autumn 2000.

Hall, J., Bisson, D. and Hare, O. (1990). The physiology of immersion. Physiotherapy. vol. 76, No. 9, September.

Harrison, RA. and Bulstrode, S. (1987). Percentage weight bearing during partial immersion in the hydrotherapy pool. Physiother Practice. 3:60-63.

Harrison, Ra., Hillma, M. and Bulstrode, S. (1992). Loading of the lower limb when walking partially immersed: Implication for clinical practice. Physiother. 78 (http:/ www.monografias.com/trabajos $12 /$ mecfluidos /mecflui.shtml): 164-166

Hejar, M., Blunt, L. (acceso 2006). OT History of Aquatics. Www.texaschildrenshospital.org/ carecenters/PhysicalMedicine/Images/ Introduction_to_Aquatic_Therapy.pdf

LAmbeck. J. (2001). Evidence based hydrotherapy: orthopedics/rheumatology: Memorias Capacitación Hidroterapia Bogotá. 13-15

LAMBeck. J. (2005). Fluidmechanic and physiology. Aquatic Therapy Course. Valens 2005.

LAMBECK, J. (2001). Hydrotherapy in adult neurology: Memorias Capacitación Hidroterapia Bogotá, abril de 2001, 9.

LAMBeck, J. (2001). The ten point-programmed as a motor learning sequence. Water specific exercises. Memorias Capacitación Hidroterapia Bogotá, abril de 2001.

Lamberck, J. and Coffey, F. (2000). The Halliwick Concept - Part 1. J. of Aquatic Physical Therapy, vol. 8 No. 2 
McNeal, citado por Poteat A (1997). EvidenceBased aquatic therapy for propioceptive retraining. Program Book Aquatic Therapy Symposium '97. Las Vegas 1997. HH98 H101.

Mecánica de fluidos. http://www.monografias.com/ trabajos 12/mecflui/mecflui.shtml. Acceso julio de 2006.

Mogollón, A. (2005). Principios de terapia acuática. Revista de la Asociación Colombiana de Fisioterapia, vol. I. 85-93.

Moscoso, F. (2005). Terapia acuática una alternativa. En Neurorrehabilitación. Revista de la Asociación Colombiana de Fisioterapia, vol. I. 107-111.

Moscoso, F. (2004). El concepto Halliwick. Memorias Simposio de Actualización en Hidroterapia. Clínica Universitaria Teletón.

Nicol, J., Schmidt-Hansberg, M. and McMillan, JY. (1979). Biomechanical principles applied to the Halliwick. Method of teaching Swimming to physically Handicapped Individuals. In Biomechanics of Swinmming. Ed. Terando Bectivs Field. Human Kinetics.

Panneso, M., Moscoso, F. (1999). Estudio del arte sobre hidroterapia. Clínica Universitaria Teletón. Chía, Colombia.

Poteat, A. (2006). Balance Training? Give patients an environment in which to fail (the pool). In: http://www.aquaticnet.com/Article\%20$\% 20 \mathrm{Balance} \% 20$ training $\% 20-\% 20$ give $\% 20$ patients\%20an\%20environment \%20in $\% 20$ which $\% 20$ to $\% 20$ fail.htm (c) Copyright 1997-2003. Acceso junio 2006.
Poteat, A. (1997). Evidence-based aquatic therapy for propioceptive retraining. Program Book of Aquatic Therapy Symposium '97. 1997. HH98 - H101.

Poteat, A. (2006). Evidence-based practice: incorporating it into aquatic therapy. http:// www.aquaticnet.com/Article\%20$\% 20$ Incorporating $\% 20$ evidence $\% 20$ based $\% 20 \mathrm{p}$ ractice $\% 20$ into $\% 20$ aquatic $\% 20$ therapy.htm Copyright 1997-2003. Acceso junio 2006.

Reid-CAmpion, M. (1997). Hydrotherapy principles and practice. Edit. Butterworth-Heinemann.

Schoedinger, P. (2000). Program book aquatic therapy symposium 2000. "Principles of Aquatic Therapy". Orlando Fl 2000 H65H72.

Schoedinger, P. (2005). Aquatic physical therapy for patients with neurologic disorders combined sections meeting 2005 New Orleans, LA Thursday, February 23-27, 2005 http://www.aquaticpt.org/events/8651.pdf Acceso febrero 2006.

Stuchiner, T. (2000). Hydrotherapy for hypotonic Babies. Program book aquatic therapy Symposium 2000. "Principles of Aquatic Therapy". Orlando Fl H209-H213.

Torpe, D., Reilly. M. (2000). The effect an aquatic. Resistive exercise program on lower extremity strength, energy expediture, functional mobility, balance and self perception in an adult with cerebral palsy: a retrospective case report. The Journal of Aquatic Physical Therapy. vol. 8, No. 2. 